\title{
Research on Teaching Reform of Japanese Pronunciation Based on Voice Visualization Software - Taking Jingdezhen Ceramic Institute as an Example
}

\author{
Yu Wu ${ }^{*}$, Danyu Wu \\ School of International Studies, Jingdezhen Ceramic Institute, Jingdezhen, 333000, China \\ * Corresponding Author: Yu Wu
}

Keywords: Japanese pronunciation, Voice visualization software, Jingdezhen Ceramic Institute

\begin{abstract}
With the teaching concept upgrading and teaching technologies development, the quality and quantity of Japanese teaching have been remarkably improved in in recent years in colleges and universities. The voice visualization software can make up for the deficiency of traditional pronunciation teaching and point out the pronunciation points clearly in the automatically generated spectrogram. The teaching practice in shows that Jingdezhen Ceramic Institute shows that the adoption of the voice visualization software can effectively enhance students' Japanese pronunciation score. We consider the test scores as the dependent variable $y$ and the utility time of voice visualization software as the independent variable $\mathrm{x}$. The polynomial fitting result of $\mathrm{x}$ and $\mathrm{y}$ is: $\mathrm{y}=$ $2.1559 x^{2}-2.8368 x+76.893$. The more time the student makes use of the voice visualization software, the good academic performance of Japanese pronunciation he will have under normal circumstances.
\end{abstract}

\section{Concept and Advantages of Voice Visualization Software}

Vision is the most efficient way for human to acquire information. The hearing sense is the following approach. Moreover, the information accessed by the combination of vision and hearing is more than the information accessed by the single sensory. The traditional pronunciation teaching only can let the student have the auditory perception, leading to an unsatisfied teaching effect. The basic function of voice visualization software is to annotate and analyse the speech signals. After recording the audio files with voice visualization software, a variety of language maps can be displayed according to requirements. It has great advantages in the voice assistant teaching, speech correction, speech autonomous learning. The voice visualization software can be downloaded free of charge. Compared with other commercial voice software, it has incomparable advantages.

Voice visualization software can complement the visual perception effectively. In terms of the vowels, when teaching the phonemes, the abstract pronunciation essentials and the similar pronunciation imitation are very difficult for students to learn. They cannot make the standard pronunciation and specify where the mistake is. The voice visualization software can provide a set of intuitive instructions through the analysis spectrogram of visual speech. The software is simple and convenient with the clear and accurate graphical feedback information. Therefore, it can be used as a wide range of voice analysis software for Japanese learners. After simple training, students can discover pronunciation problems and correct them by using their own software. Teachers can also put forward some specific solutions to improve the teaching effect and efficiency according to the results of students' pronunciation assessment by the voice visualization software.

\section{Application Effects of Voice Visualization Software in Japanese Pronunciation Teaching}

\subsection{Comparison between the Class using Voice Visualization Software and the Traditional Class}

We take the Japanese major students in Jingdezhen Ceramic Institute as the research object in this study. There are 29 students in class 1 of the Japanese major and there are 30 students in class 2 of the 
Japanese major. We adopt the voice visualization software in class 1 and the traditional teaching mode in class 2 to teach the Japanese pronunciation. The learning process is pluralistic and the students' academic performance is influenced by many factors. We should ensure all the other conditions are same except the teaching model. The training program, teaching materials, teachers and the final exams are all the same in class 1 and class 2 . The scores of the final examination of class 1 (using the voice visualization software) and class 2 (traditional model class) are shown in Table 1. The number represents the identification number of every student in each class.

Table 1. Japanese pronunciation scores of class 1 and class 2

\begin{tabular}{|c|c|c|c|}
\hline \multicolumn{2}{|c|}{ Class 1} & \multicolumn{2}{|c|}{ Class 2} \\
\hline Number & Score & Number & Score \\
\hline 001 & 90 & 001 & 88 \\
\hline 002 & 88 & 002 & 86 \\
\hline 003 & 75 & 003 & 77 \\
\hline 004 & 78 & 004 & 80 \\
\hline 005 & 90 & 005 & 66 \\
\hline 006 & 83 & 006 & 73 \\
\hline 007 & 92 & 007 & 90 \\
\hline 008 & 73 & 008 & 82 \\
\hline 009 & 78 & 009 & 80 \\
\hline 010 & 77 & 010 & 77 \\
\hline 011 & 82 & 011 & 77 \\
\hline 012 & 78 & 012 & 82 \\
\hline 013 & 79 & 013 & 71 \\
\hline 014 & 72 & 014 & 80 \\
\hline 015 & 77 & 015 & 70 \\
\hline 016 & 93 & 016 & 72 \\
\hline 017 & 72 & 017 & 75 \\
\hline 018 & 81 & 018 & 69 \\
\hline 019 & 78 & 019 & 87 \\
\hline 020 & 79 & 020 & 90 \\
\hline 021 & 92 & 021 & 73 \\
\hline 022 & 78 & 022 & 89 \\
\hline 023 & 84 & 023 & 74 \\
\hline 024 & 89 & 024 & 66 \\
\hline 025 & 74 & 025 & 69 \\
\hline 026 & 71 & 026 & 81 \\
\hline 027 & 73 & 027 & 67 \\
\hline 028 & 90 & 028 & 71 \\
\hline 029 & 85 & 029 & 76 \\
\hline & & 030 & 76 \\
\hline
\end{tabular}

We calculate the mean and standard deviation of the scores of class 1 and class 2 . The results are shown in Table 2.

Table 2. Mean and standard deviation of scores in class 1 and class 2

\begin{tabular}{|l|r|r|}
\hline & \multicolumn{1}{|c|}{ Class 1 } & \multicolumn{1}{l|}{ Class 2 } \\
\hline Mean & 81.1 & 77.1 \\
\hline Standard Deviation & 6.9 & 7.3 \\
\hline
\end{tabular}

From Table 2, we can know that the mean score of the thirty students taught in the traditional 
model in Class 2 is 77.1. However, the mean score of the twenty-nine students adopting the voice visualization software in Class 1 is 81.1. Obviously, the teaching performance using the voice visualization software is better than that of the traditional model. In terms of standard deviation, the standard deviation value using the voice visualization software is lower than that of the traditional class, which shows that the voice visualization software can effectively reduce the instability of the test scores.

\subsection{Relationship between Score and Utility Time of Voice Visualization Software}

Aimed at studying the relationship between the utility time of voice visualization software and the Japanese pronunciation scores, the author launched a relevant survey. We combine the utility time in every day and the final examination score to get the Table 3.

Table 3. The utility time of voice visualization software and Japanese pronunciation scores of every student

\begin{tabular}{|c|c|c|}
\hline \multicolumn{3}{|c|}{ Class 1} \\
\hline Number & Score & Time \\
\hline 001 & 90 & 2.5 \\
\hline 002 & 88 & 3.0 \\
\hline 003 & 75 & 0.8 \\
\hline 004 & 78 & 2.5 \\
\hline 005 & 90 & 1.7 \\
\hline 006 & 83 & 2.9 \\
\hline 007 & 92 & 3.0 \\
\hline 008 & 73 & 0.5 \\
\hline 009 & 78 & 0.5 \\
\hline 010 & 77 & 0.7 \\
\hline 011 & 82 & 2.0 \\
\hline 012 & 78 & 1.1 \\
\hline 013 & 79 & 1.2 \\
\hline 014 & 72 & 2.5 \\
\hline 015 & 77 & 1.9 \\
\hline 016 & 93 & 2.9 \\
\hline 017 & 72 & 1.3 \\
\hline 018 & 81 & 2.5 \\
\hline 019 & 78 & 2.2 \\
\hline 020 & 79 & 0.9 \\
\hline 021 & 92 & 3.5 \\
\hline 022 & 78 & 1.5 \\
\hline 023 & 84 & 2.0 \\
\hline 024 & 89 & 3.0 \\
\hline 025 & 74 & 0.5 \\
\hline 026 & 71 & 2.3 \\
\hline 027 & 73 & 1.5 \\
\hline 028 & 90 & 2.5 \\
\hline 029 & 85 & 2.5 \\
\hline
\end{tabular}

We draw the scatter diagram of utility time of voice visualization software and Japanese pronunciation score of every student, and then do the polynomial fitting. The results are shown in Figure 1. 


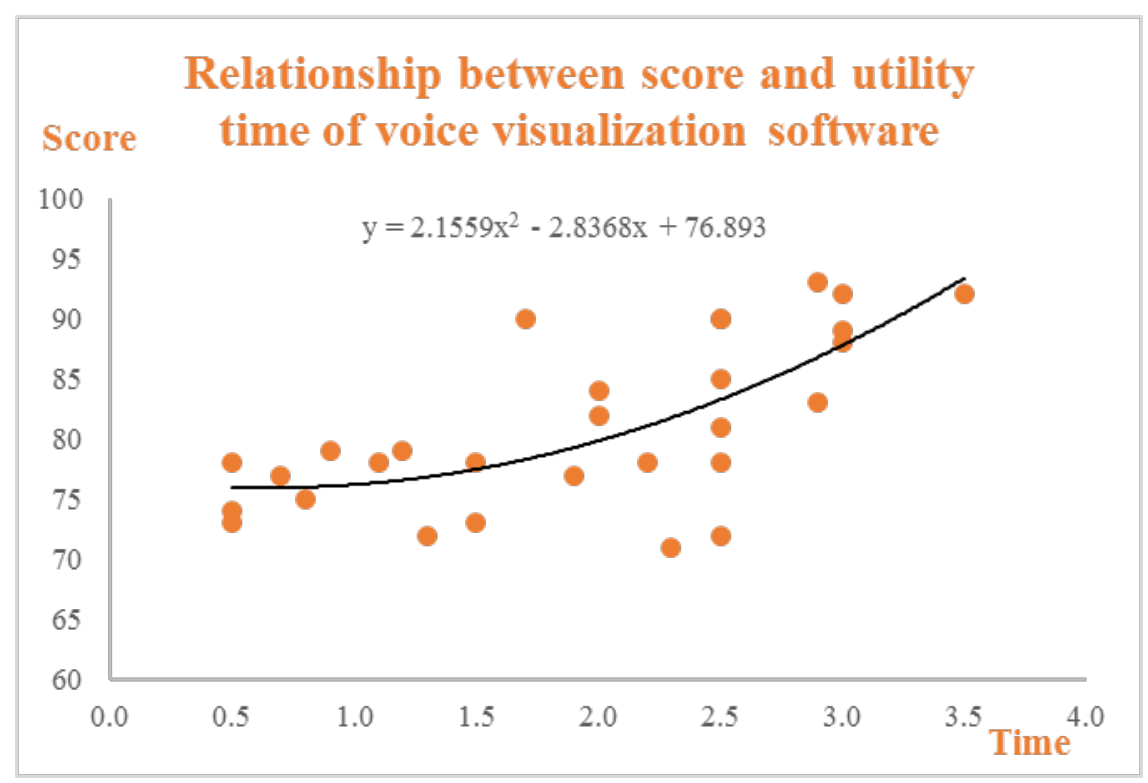

Figure 1. Relationship between score and utility time of voice visualization software

When we consider the test scores as the dependent variable y and the utility time of voice visualization software as the independent variable $\mathrm{x}$. The polynomial relationship between $\mathrm{x}$ and $\mathrm{y}$ is:

$$
y=2.1559 x^{2}-2.8368 x+76.893
$$

According to the mathematical knowledge, in the interval of $[0.66,+\infty)$, the score of Japanese pronunciation will increase with the prolong of the utility time of voice visualization software in general.

That is to say, in the interval of $[0.66,+\infty)$, the more time to use the voice visualization software, the better test results will be obtained. As the 0.66 hour can be seen as the minimum utility time for students to use the software, we can say that the more time to use the voice visualization software, the good academic performance the students have under normal circumstances.

\section{Conclusion}

This paper explores the difficulties of Japanese pronunciation teaching and verifies the important role of the voice visualization software through empirical study. The adoption of the voice visualization software can effectively enhance students' Japanese pronunciation scores by making up the visual shortage of the traditional pronunciation teaching model. The teaching practice shows when the utility time of voice visualization software is in the interval of $[0.66,+\infty)$, the score of Japanese pronunciation will increase with the prolong of the utility time of voice visualization software in general.

\section{References}

[1] Mei W. Research on Japanese Teaching Based on ASSURE Model: A Case Study on Japanese Dictation[J]. The Science Education Article Collects, 2014, 11: 057.

[2] Yuan Q U. Introduction of Chinese Culture into College Japanese Teaching[J]. Journal of Inner Mongolia Normal University (Philosophy and Social Sciences Edition), 2013, 1: 020.

[3] Lin Q I. Japanese Teaching Should Combine Direct Method and Indirect Method[J]. Journal of Dalian Education University, 2013, 2: 014.

[4] Yan H. The Inspiration of Effective Teaching Theory on Japanese Teaching of Higher Vocational Colleges[J]. Journal of Huaihai Institute of Technology (Humanities \& Social Sciences Edition), 2013, 7: 021. 
[5] Lei S. A Brief Discussion on the Reform of Business Japanese Teaching Based on the Requirement of Talents for Foreign Trade[J]. The Science Education Article Collects, 2013, 10: 077.

[6] Dongmei B, Yong Y. Research on Cultural Introduction in the Process of Chinese and Japanese Cultural Interpenetration in Japanese Teaching[J]. Journal of Xuchang University, 2013, 1: 038.

[7] Hui R, Jiang W. Application of the" Can-do Mode" of JF Japanese Education Standards in Language Teaching Practice[J]. The Science Education Article Collects, 2014, 1: 081.

[8] Yan X, Jian C, Nian-nian L, et al. Construction of personnel training mode and the reform of teaching practice of business Japanese specialty_—Research Report of Japanese Enterprises[J]. Journal of Jiamusi Education Institute, 2014, 2: 015. 